\title{
The self-efficacy for therapeutic use of self- questionnaire (SETUS): psychometric properties of the English version
}

\author{
Farzaneh Yazdani \\ Oxford Brookes University Faculty of Health and Life Sciences, Oxford, UK \\ Tore Bonsaksen \\ Department of Health and Nursing, Inland Norway University of Applied Sciences, Elverum, Norway and Faculty of Health Studies, \\ VID Specialized University, Sandnes, Norway \\ Dave Roberts and Ka Yan Hess \\ Oxford Brookes University Faculty of Health and Life Sciences, Oxford, UK, and \\ Samaneh Karamali Esmaili \\ Department of Occupational Therapy, School of Rehabilitation Sciences, Iran University of Medical Sciences, Tehran, Iran
}

\begin{abstract}
Purpose - The purpose of this paper is to investigate psychometric properties of the Self-Efficacy for Therapeutic Use of Self (SETUS) scales, a questionnaire based on the Intentional Relationship model, and to investigate the factor structure and internal consistency of the English version of three-part SETUS questionnaire in occupational therapy students.

Design/methodology/approach - The sample of this cross-sectional study included 155 students with age range 18-30 years, of which $95 \%$ were women. Principal Components Analysis (PCA) was performed on the questionnaire scales, including the Self-Efficacy for Therapeutic Mode Use (SETMU), Self-Efficacy for Recognizing Interpersonal Characteristics (SERIC) and Self-Efficacy for Managing Interpersonal Events (SEMIE). The internal consistencies were calculated. Pearson correlation analysis was used to evaluate the strength of correlation among the scales.

Findings - The PCA confirmed that the items of each of the three proposed scales loaded strongly on one factor (self-efficacy for three factors of therapeutic mode use, recognizing interpersonal characteristics and managing interpersonal events). The Cronbach's alpha for the SETMU, SERIC and SEMIE was $0.85,0.95$ and 0.96 , respectively. The three scales significantly inter-correlated strongly ( $r$ ranging $0.74-0.83$, all $p<0.001$ ).

Originality/value - The SETUS questionnaire comprises three valid and reliable scales. It can be used by occupational therapy supervisors as a means to reflect on students' self-efficacy in components of therapeutic use of self.
\end{abstract}

Keywords Reliability, Self-efficacy, Factor analysis, Occupational therapy, Therapeutic use of self

Paper type Research paper

\section{Introduction}

Self-efficacy refers to people's perceptions of their effectiveness in using a personal ability to achieve the optimal results (Bandura, 1997). Higher self-efficacy leads to more motivation to face challenges and more effort to achieve the desired goal. As a range of studies have shown, having self-efficacy to perform a task or occupation increases the likelihood of success (Nouwen et al., 2009; Scholz et al., 2009; Zeegers, 2004).

Self-efficacy is an essential element for health-care practitioners to perform a wide range of professional roles and tasks. Herold et al.'s (2005) study demonstrated that rehabilitation professionals including occupational therapists, physical therapists and speech-language pathologists, did not

The current issue and full text archive of this journal is available on Emerald Insight at: https://www.emerald.com/insight/2398-8819.htm

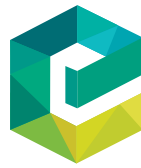

Irish Journal of Occupational Therapy

49/1 (2021) 21-27

Emerald Publishing Limited [ISSN 2398-8819]

[DOI 10.1108/IJOT-10-2020-0015] have sufficient self-efficacy in their professional roles. They investigated self-efficacy for specific work tasks including selfefficacy in practice based on evidence, advocacy, developing emerging markets, practice based on functional outcomes, pursuing lifelong learning and using innovative approaches to

(C) Farzaneh Yazdani, Tore Bonsaksen, Dave Roberts, Ka Yan Hess and Samaneh Karamali Esmaili. Published in Irish fournal of Occupational Therapy. Published by Emerald Publishing Limited. This article is published under the Creative Commons Attribution (CC BY 4.0) licence. Anyone may reproduce, distribute, translate and create derivative works of this article (for both commercial and non-commercial purposes), subject to full attribution to the original publication and authors. The full terms of this licence maybe seen at http://creativecommons.org/licences/by/4.0/legalcode

Participants in the present study, occupational therapy students, are appreciated. This study did not receive funding from any source.

Declaration of Interest statement: There was no conflict of interests in the study.

Received 27 October 2020

Revised 19 January 2021

13 March 2021

Accepted 1 April 2021 
reach organizational goals. In addition to self-efficacy in these areas of work performances, self-efficacy for managing a specific disorder (Burch, 2008) or practice setting (Chandler, 2008; Vax et al., 2012) have been studied. However, in addition to work performance and knowledge, the client-therapist relationship is a key component to success in the professional role and self-efficacy in this regard is critical.

An appropriate therapeutic relationship can facilitate the process of therapy. In the professions such as occupational therapy, interpersonal aspects of therapy play a vital role to help therapists to achieve positive treatment outcomes (Pan and Liu, 2016). As a principle in occupational therapy, a successful therapeutic relationship is contingent on the therapist's therapeutic use of self (Punwar and Peloquin, 2000; Taylor, 2008). The therapeutic use of self is a practitioner's conscious efforts in using his/her personality, judgments and perceptions to optimize interactions with clients (Punwar and Peloquin, 2000; Gillen, 2014). If the therapist perceives self-efficacy in him/her therapeutic use of self, he/she can have the optimal practice, achieve therapeutic goals and achieve client satisfaction (Taylor, 2008).

The Intentional Relationship Model (IRM; Taylor, 2008) is the first conceptual practice model that systematically identifies interpersonal aspects of therapy and facilitates therapists' understanding of the impact of therapeutic use of self on the treatment process. It directs attention to the client's interpersonal characteristics (client's interpersonal behaviours when experiencing stressful circumstances), potentially disruptive interpersonal events (events occurring during client-therapist interaction that need handling) and therapist's relationship approach with the client (the therapeutic mode that is used). The therapeutic modes are six specific relationship styles with clients. They comprise the following modes: advocating (helping the client in accessing necessary resources), collaborating (including the client in all stages of the therapy), empathizing (making efforts to understand the client's feelings and inner experiences), encouraging (applauding and cheering the client's performance), instructing (playing a role of teacher and guiding the client) and problem-solving (analyzing the client's problems and finding solution) (Taylor, 2008; Bonsaksen et al., 2013).

The IRM emphasizes four components of the therapeutic relationship and their interaction with each other, including the client, the therapist, interpersonal events and the occupation as goal and means of intervention (Taylor, 2014; Taylor, 2008). The therapist must consciously and flexibly use the appropriate mode and adjust it moment-by-moment in treatment. To do this, he/ she must regularly review his/her mode and reflect on his/her own practice to enhance it. In addition to the therapeutic mode, the therapist's ability to recognize the interpersonal characteristics of the client and manage the challenges encountered in the treatment session will greatly help to enhance clinical practice. More importantly, the therapist's perceptions about effectiveness in using these three personal abilities (therapeutic mode, recognizing the interpersonal characteristics and managing the interpersonal events) shape self-efficacy in therapeutic use of self. To accurately evaluate these areas and to apply the IRM in practice, the Self-Efficacy for Therapeutic Use of Self Questionnaire (SETUS) was developed by Yazdani and Tune (2016).
According to the three aspects of therapeutic use of self in the IRM that mentioned above, the SETUS questionnaire consists of three scales to assess different aspects of therapeutic use of self. These include the Self-Efficacy for Therapeutic Mode Use (SETMU), the Self-Efficacy for Recognizing Interpersonal Characteristics (SERIC) and the Self-Efficacy for Managing Interpersonal Events (SEMIE) scales. The Norwegian versions of the scales were prepared and psychometrically evaluated (Bonsaksen and Carstensen, 2018; Bonsaksen et al., 2018; Ritter et al., 2018). These studies demonstrated that each of the scales had a one-factor structure and that the internal consistency of the items comprising each scale, was high (0.82 for SETMU; Bonsaksen and Carstensen, 2018) or very high (0.96 for SERIC and 0.94 for SEMIE; Bonsaksen et al., 2018; Ritter et al., 2018). Other studies have been conducted using the Norwegian version of the SETUS questionnaire. Opseth et al. (2017), Ritter et al. (2017), Schwank et al. (2018), Hussain et al. (2018), Fan et al. (2020a), and Fan et al. (2020b) used this questionnaire in their studies to assess self-efficacy for therapeutic use of self in Norwegian occupational therapy students.

Having a valid and reliable tool to assess self-efficacy for therapeutic use of self provides a self-appraisal opportunity for students and practitioners. To achieve this, it is necessary to conduct psychometric studies on the instruments. These could include construct, concurrent, predictive validity or intra- and inter-rater and test-retest reliability (Kielhofner and Fossey, 2006). Having a tool with these characteristics can be even more important for students than practitioners because therapeutic use of self may be difficult to learn. A recent research study shows that training and empowering of occupational therapy students to apply IRM model in practice increases students' self-efficacy in therapeutic use of self (Fan et al., 2020a). Taylor et al. (2009) found that about half of occupational therapy students graduating from university feel they have not received adequate training for the therapeutic relationship and use of self; also, students might undervalue their abilities in therapeutic use of self (Davidson, 2011), and they are more likely to understand its importance as their level of experience increases (Taylor et al., 2009). In Davidson's (2011) mixed-method study of 39 entry level occupational therapy programs, although therapeutic use of self was considered critical for entry level education, its content and teaching method were not well defined. The SETUS questionnaire can help supervisors to monitor students' self-perception about therapeutic use of self, provide them with feedback and help them to make better plans for teaching the necessary skills and knowledge. This instrument also facilitates the application of IRM in practice because, rather than simply encouraging a general reflection on practice, it reviews the concepts of the IRM item-by-item, so the student can have a detailed self-reflection.

While comprehensive studies have been done on the Norwegian version of the SETUS questionnaire, the psychometric properties of the SETMU were also investigated in a small pilot study of English/Iranian occupational therapists (Yazdani and Bonsaksen, 2019). Similar to the Norwegian studies, this study showed that the SETMU items had a one-factor structure with high internal consistency between the individual items (Cronbach's $\alpha=0.83$ ). However, psychometric information related to the English version of all parts of the instrument (SETUS), with an appropriately large 
sample, is needed. The purpose of this study is to explore the factor structure and internal consistency of the English version of three parts of the SETUS questionnaire: the SETMU, SERIC and SEMIE, in occupational therapy students.

\section{Materials and methods}

This research was a descriptive cross-sectional study to determine psychometric properties of the three-part SETUS questionnaire.

\section{Participants}

The participants were selected by convenience sampling. They were 155 occupational therapy students. The age range was 18-30 years, of which $95 \%$ were women. The response rate was $67 \%$. Students were taught a module on therapeutic use of self and they completed the SETUS questionnaire after having provided informed consent to participate in the study. Of these, $22 \%(n=34)$ were postgraduate $(12 \%$ first- and $10 \%$ secondyear) and $78 \%(n=121)$ were undergraduate (52\% first-, $30 \%$ second- and $18 \%$ third-year) students. Because of the power relationship between the researcher (first author) and students and potential identification of the students in a small group, no demographic information was collected for the study sample, to ensure that individual participants felt confident that they could not be identified. This was a requirement of the University ethics committee.

\section{Instrument}

The instrument that was investigated in this study was the SETUS. Yazdani and Tune (2016) developed this tool in the UK based on a combination of Bandura's social cognitive theory and Taylor's model of the therapeutic relationship in occupational therapy (IRM). The SETUS instrument measures therapists' self-efficacy in three different aspects of therapeutic use of self, according to Taylor's (2008) outline in the IRM of the essential aspects of relating in therapeutic contexts. Consistent with these three aspects, the SETUS includes three scales: the SETMU scale with 6 items assesses perceived self-efficacy for using the therapeutic modes in relationships with clients, the SERIC scale with 12 items that helps therapists to assess their self-efficacy in recognizing interpersonal characteristics of clients, and the SEMIE scale with 11 items that helps to determine therapists' self-efficacy in managing the interpersonal events inevitably occurring during therapy. The therapist/student is asked to determine perceived self-efficacy on a ten-point scale for each item (from 1 = lowest possible self-efficacy to $10=$ highest possible self-efficacy); therefore, minimum scores for the SETMU, SERIC and SEMIE scales will be 6, 12 and 11, and the maximum scores will be 60,120 and 110 , respectively.

\section{Procedure}

The study was conducted at the Oxford Brookes University. The participants were occupational therapy students who had successfully passed "the module of therapeutic use of self" in the relevant exam. This module comprised three hours of IRM introduction and $15 \mathrm{~h}$ of interactive seminars. The three focal topics of IRM covered in these seminars were: therapist characteristics and skills (building rapport, communication, modes' characteristics, precautions, strategies and applications), client's interpersonal characteristics (challenging behaviour, situational and enduring interpersonal characteristics) and inevitable interpersonal events of therapy. In this module, the students were set to do activities including practicing scenarios, filming their own role playing, reflecting on their own actions and providing feedback to the others.

After passing the module exam, the students filled in the three-part SETUS questionnaire. The questionnaires were distributed among them by one of the university staff and anyone who was volunteered participated in the study.

\section{Data analysis}

Descriptive analysis was performed using means and standard deviations and the scale score range and interquartile range was calculated for each scale. To examine latent factors in each part of the SETUS questionnaire, Principal Component Analysis (PCA) was performed. To assess whether the data were appropriate for factorization, The Bartlett's Test of Sphericity and Kaiser-Meyer-Olkin (KMO) Measure of Sampling adequacy (should be greater than 0.6) were used (Kaiser, 1974). Visual inspection of the scree-plots, assessment of the eigenvalues and assessment of the variance explained by the factors were used to determine factor extraction. Factor loadings $>0.40$ were considered strong (Field, 2005). The internal consistency (scale reliability) was measured with Cronbach's alpha $(>0.70=$ good $)$ and inter-item correlations $(>0.20=$ good $)($ Cortina, 1993). The data were analyzed by IBM SPSS version 24 (IBM Corporation, 2016). Statistical significance was considered as $p<0.05$.

\section{Ethics}

Ethical approval for the study was obtained from the Faculty of Health and Life Sciences at Oxford Brookes University and the Health Research Authority (approval number 2014/38). The module of training in therapeutic use of self was a part of the education program of students but for completing the SETMU the first author informed them about the aim of the study and provided a written consent form. The students were assured that participation in the research was voluntary and their information would remain confidential.

\section{Results}

The sample consisted of 155 occupational therapy students. A number of students responded to one or two scales of the questionnaire incompletely; so, 10 respondents were removed from the analysis of SETMU, 14 were removed from the analysis of SERIC, and 19 were removed from the analysis of SEMIE, so, 145 SETMU, 136 SEMIE and 141 SERIC were analyzed. Descriptive statistics are reported in Table 1 . The participants perceived similar self-efficacy on all three scales; however, the total mean score of the SEMIE was lower than the others.

To determine the appropriateness of the data set for factor analysis and component extraction, the KMO and Bartlett's Test of Sphericity were calculated. For the SETMU scale, the KMO value was found to be 0.78 and Bartlett's test was statistically significant $(p<0.01)$; for the SERIC scale, the KMO value was found to be 0.92 and Bartlett's test was 
Table 1 Score range, interquartile range and mean scale scores

\begin{tabular}{lcccc}
\hline Scale & $\boldsymbol{n}$ & Score range (min-max) & Interquartile range (IQR) $^{*}$ & Mean scale Score (SD $^{* * * *}$ ) \\
\hline SETMU $^{*}$ & 145 & $2.83-9.50$ & 1.50 & $6.99(1.25)$ \\
SERIC $^{* *}$ & 141 & $1.83-10.00$ & 1.75 & $6.58(1.36)$ \\
SEMIE $^{* * *}$ & 136 & $2.45-10.00$ & 1.91 & $6.45(1.46)$
\end{tabular}

Notes: ${ }^{* * * *}$ Standard deviation; *the Self-Efficacy for Therapeutic Mode Use; ${ }^{* *}$ the Self-Efficacy for Recognizing Interpersonal Characteristics; ${ }^{* * *}$ the SelfEfficacy for Managing Interpersonal Events

statistically significant $(p<0.01)$; and for the SEMIE scale, the KMO value was found to be 0.93 and Bartlett's test was statistically significant $(p<0.01)$.

The Eigenvalue $(\lambda)$ estimates, a visual inspection of the scree-plots, and the explained variance by the factors determined the number of extracted factors. In each of the scales, only one factor had Eigenvalue $>1$ and that factor explained between 57\% (SETMU) and 71\% (SEMIE) of the variables' variance proportions (Tables 2-4). For the SETMU scale, Factor $2 \lambda=0.84$ explained $14.6 \%$ of the variance; for the SERIC scale, Factor $2 \lambda=0.89$ explained $7.44 \%$ of the variance; and for the SEMIE scale, Factor $2 \lambda=0.76$ explained $6.98 \%$ of the variance. Thus, only one factor was extracted from each of the SETMU, SERIC and SEMIE scale variables, respectively.

All items comprising the SETMU scale loaded strongly on the single latent factor (factor loadings ranging between 0.69 and 0.84). Similarly, all items on the SERIC scale loaded strongly on the single latent factor (factor loadings ranging between 0.73 and 0.89), as did all items on the SEMIE scale (factor loadings ranging between 0.78 and 0.90 ).

The internal consistency of the three scales is reported in Tables 2-4. For the three scales, Cronbach's coefficient alpha ranged between 0.85 (SETMU) and 0.96 (SEMIE) and the mean inter-item correlations ranged between 0.49 (SETMU) and 0.68 (SEMIE), indicating very high internal consistency for each of the three scales.
Strong correlations $(r$ ranging between 0.74 and 0.83 , all $p<$ 0.001) were demonstrated between each of the scales of the SETUS questionnaire. These results indicate that students with higher scores on one scale also had higher scores on the others and vice versa.

\section{Discussion}

The present study was conducted to evaluate construct validity and internal consistency of the three scales of the SETUS

Table 2 Factor loadings, communalities, eigenvalues and internal consistency estimates related to the SETMU

\begin{tabular}{lcc}
\hline Item & Factor 1 loadings & Communalities \\
\hline Collaborating & 0.835 & 0.698 \\
Problem solving & 0.776 & 0.602 \\
Instructing & 0.765 & 0.585 \\
Encouraging & 0.748 & 0.560 \\
Empathizing & 0.714 & 0.510 \\
Advocating & 0.692 & 0.479 \\
Eigenvalue & 3.43 & - \\
Explained variance & $57.23 \%$ & - \\
Cronbach's $\alpha$ & 0.85 & - \\
Mean inter-item correlation & 0.49 & - \\
Note: SETMU is Self-Efficacy for Therapeutic Mode Use & \\
\hline
\end{tabular}

Table 3 Factor loadings, communalities, eigenvalues and internal consistency estimates related to the SERIC

\begin{tabular}{lcc}
\hline Item & Factor 1 loadings & Communalities \\
\hline Preference for communication style & 0.725 & 0.525 \\
Capacity for trust & 0.858 & 0.737 \\
Need for control & 0.778 \\
Capacity to assert needs & 0.855 \\
Response to change or challenge & 0.829 \\
Affect & 0.783 \\
Predisposition to giving feedback & 0.879 \\
Predisposition to receiving feedback & 0.885 \\
Response to human diversity & 0.794 \\
Orientation toward relating & 0.761 \\
Preference for touch & 0.767 \\
Capacity for reciprocity & 0.812 \\
Eigenvalue & 7.84 \\
Explained variance & $65.92 \%$ \\
Cronbach's $\alpha$ & 0.95 \\
Mean inter-item correlation & 0.63 \\
Note: SERIC is Self-Efficacy for Recognizing Interpersonal Characteristics & & 0.687 \\
\hline
\end{tabular}


Table 4 Factor loadings, communalities, eigenvalues and internal consistency estimates related to the SEMIE

\begin{tabular}{lcc}
\hline Item & Factor 1 loadings & Communalities \\
\hline Expression of strong emotion & 0.826 & 0.682 \\
Intimate self-disclosures & 0.803 & 0.645 \\
Power dilemmas & 0.857 & 0.734 \\
Non-verbal cues & 0.850 & 0.723 \\
Crisis points & 0.866 & 0.749 \\
Resistance and reluctance & 0.877 & 0.769 \\
Boundary testing & 0.858 \\
Empathic breaks & 0.837 \\
Emotionally charged tasks and situations & 0.904 \\
Limitations of therapy & 0.775 \\
Contextual inconsistencies & 0.827 \\
Eigenvalue & 7.91 \\
Explained variance & $71.29 \%$ \\
Cronbach's $\alpha$ & 0.96 \\
Mean inter-item correlation & 0.68 \\
Note: SEMIE is Self-Efficacy for Managing Interpersonal Events & \\
\hline
\end{tabular}

questionnaire. According to the results, the three scales comprising the SETUS questionnaire all have good psychometric properties to assess self-efficacy for therapeutic use of self among occupational therapy students. Examining each scale individually showed that each scale of the SETUS questionnaire measures a unidimensional construct.

The findings of this psychometric analysis are generally consistent with the literature. Fan et al. (2020b) using itemresponse theory on the Norwegian version of SETUS questionnaire indicated that each of the three scales represented one latent trait of self-efficacy. Also, the studies using classical test theory on the Norwegian versions of the SETMU, SERIC and SEMIE, confirmed their unidimensionality (Bonsaksen and Carstensen, 2018; Bonsaksen et al., 2018; Ritter et al., 2018). Other self-efficacy assessment tools such as the General SelfEfficacy Scale have generally showed one-factor structure (Scholz et al., 2002). However, the three scales were strongly correlated, indicating that they assess interrelated constructs. Positive associations between the scales would be in line with IRM theory (Taylor, 2008), which underpins the structure of the three-part SETUS questionnaire.

The findings related to reliability demonstrated that the internal consistency of each of the scales in the SETUS questionnaire is satisfactory. The 6 items in the SETMU, 12 items in the SERIC, and 11 items in the SEMIE showed a high level of internal consistency with each other. These results are consistent with the findings pertaining to the Norwegian versions of SETMU, SERIC and SEMIE scales. The Cronbach's alpha for the SETMU (0.85) is close to Bonsaksen and Carstensen's (2018) study (0.82); the value for the SERIC in this study (0.95) is close to Ritter et al. 's (2018) study (0.96); and Cronbach's alpha for the SEMIE in this study (0.96) is close to Bonsaksen et al.'s (2018) study (0.94). It can be concluded that the scales of the SETUS questionnaire have very high internal consistency.

The participants' responses to the questionnaire showed that they generally used the full range of responses provided for this instrument. According to Table 1, the participants used all scores from 1 to 10 (except that score 1 was not used in the SETMU). A ten-point rating scale allows for great sensitivity and the possibility of differentiating between fairly similar responses, and the prospects of reducing the number of response categories has been discussed previously (Bonsaksen and Carstensen, 2018; Fan et al., 2020). However, given the participants' response pattern, it appears this level of scale sensitivity is needed. More categories on the rating scale increase its sensitivity (Myers, 1999). Thus, a tenpoint Likert scale seems appropriate for measuring self-efficacy for therapeutic use of self; especially when evaluating self-efficacy in students who may be from different academic years and have a variety of experiences in direct clinical practice with clients. On the other hand, Streiner et al. (2015) suggested that the best rating scale may be a seven-point scale. Chen et al. (2001) identified a five-point Likert scale for the new general self-efficacy (NGSE) scale. Somewhat contradictory findings in this literature indicate that further research in this area is warranted.

There can be many recommendations for future studies on the SETUS questionnaire and its three scales. Psychometric studies that measure other types of validity and reliability are needed. Research on the SETUS questionnaire using an itemresponse theory approach (Rasch analysis), reflecting what was conducted with the Norwegian version (Fan et al., 2020b), is suggested. Assessing self-efficacy for therapeutic use of self in relation to different levels of occupational therapists' experience would be helpful. It is quite clear that increasing experience increases self-efficacy for therapeutic use of self (Fan et al., 2020a); however, the impact of teaching therapeutic use of self in the education program for occupational therapy students should be investigated. The SETUS tool may be used to measure possible outcomes of interventions or training, by comparing each individual's sum scores for each of the scales before and after the training. Furthermore, because of the complexity and variety of occupational therapy practice in different work settings, self-efficacy for therapeutic use of self may be compared between therapists in different fields of practice.

\section{Methodological considerations/limitations}

The recommendations for the sample size to be used in factor analysis vary widely. In Knapp and Brown's (1995) study, the 
proposed ratios were between three and 40-50 subjects for each item. In conclusion, they recommended a 5:1 ratio between subjects and items. Given that we have treated the three scales separately, we have no more than 12 items in any analysis. Therefore, in this study, responses from no less than 136 participants were analyzed in relation to no more than 12 items (Table 1), providing at last a 11:1 ratio between subjects and items in the analyses. Therefore, the sample was considered to be of sufficient size.

A major limitation in the present study was the sampling method. The study population included occupational therapy students from one university who were selected by convenience and self-recruitment. Also, it was possible that the students would have a social desirability bias in responding to the SETUS. To avoid this bias, individual demographic data was not collected so that participants could not be identified.

\section{Conclusion}

The purpose of this study was to investigate the factor structure and internal consistency of the English version of SETUS questionnaire in occupational therapy students. The questionnaire was shown to comprise three valid and reliable assessment scales that reflect students' self-efficacy in three different yet interrelated aspects of therapeutic use of self. Each scale was shown to have a one-factor structure with very good internal consistency between individual items. Following training related to the therapeutic use of self, students' self-efficacy for therapeutic use of self could be regularly evaluated by using the SETUS questionnaire. The SETUS questionnaire may inform supervisors about students' self-efficacy in this area and can be used as a self-reflection tool for students.

\section{References}

Bandura, A. (1997), Self-Efficacy: The Exercise of Control, WH Freeman and Company, New York, NY.

Bonsaksen, T. and Carstensen, T. (2018), "Psychometric properties of the Norwegian Self-Efficacy for therapeutic mode use (N-SETMU)", Scandinavian Fournal of Occupational Therapy, Vol. 25 No. 6, pp. 475-480, doi: 10.1080/ 11038128.2017.1316421.

Bonsaksen, T., Vøllestad, K. and Taylor, R.R. (2013), "The intentional relationship Model - Use of the therapeutic relationship in occupational therapy practice", Ergoterapeuten, Vol. 56 No. 5, pp. 26-31.

Bonsaksen, T., Yazdani, F., Ellingham, B. and Carstensen, T. (2018), "Psychometric properties of the Norwegian Self Efficacy for managing interpersonal events (N-SEMIE)", Ergoscience, pp. 1-20.

Burch, A. (2008), "Health care providers' knowledge, attitudes, and self-efficacy for working with patients with spinal cord injury who have diverse sexual orientations", Physical Therapy, Vol. 88 No. 2, pp. 191-198, doi: 10.2522/ ptj. 20060188.

Chandler, B.E. (2008), "An examination of factors that affect occupational therapists' self efficacy related to working with students who have emotional disturbance", $\mathrm{PhD}$, TUI University, ERIC, available at: https://eric.ed.gov/? id=ED 527297
Chen, G., Gully, S.M. and Eden, D. (2001), "Validation of a new general self-efficacy scale", Organizational Research Methods, Vol. 4 No. 1, pp. 62-83, doi: 10.1177/109442810141004.

Cortina, J.M. (1993), "What is coefficient alpha? An examination of theory and applications", fournal of Applied Psychology, Vol. 78 No. 1, pp. 98-104, doi: 10.1037/00219010.78.1.98.

Davidson, D.A. (2011), "Therapeutic use of self in academic education: a mixed-methods study", Occupational Therapy in Mental Health, Vol. 27 No. 1, pp. 87-102, doi: 10.1080/ $0164212 \mathrm{X} .2011 .543966$.

Fan, C.W., Yazdani, F., Carstensen, T. and Bonsaksen, T. (2020a), "Rasch analysis of the Self-Efficacy for therapeutic use of self questionnaire in Norwegian occupational therapy students", Scandinavian fournal of Occupational Therapy, doi: 10.1080/11038128.2020.1726453.

Fan, C.W., Carstensen, T., Småstuen, M.C., Yazdani, F., Ellingham, B. and Bonsaksen, T. (2020b), "Occupational therapy students' self-efficacy for therapeutic use of self: development and associated factors", fournal of Occupational Therapy Education, Vol. 4 No. 1, pp. 1-13, doi: 10.26681/ jote.2020.040103.

Gillen, G. (2014), "Occupational therapy interventions for individuals”, in Schell, B., Gillen, G. and Scaffa, M. (Eds), Willard and Spackman's Occupational Therapy, Lippincott Williams \& Wilkins, Philadelphia, pp. 322-341.

Herold, C., Bennett, O. and Costello, P. (2005), "Selfefficacy for career development: a study of occupational therapists, physical therapists, and speech-language pathologists in the United States", Fournal of Vocational Education \& Training, Vol. 57 No. 1, pp. 61-80, doi: 10.1080/13636820500200275.

Hussain, R.A., Carstensen, T., Yazdani, F., Ellingham, B. and Bonsaksen, T. (2018), "Short-term changes in occupational therapy students' self-efficacy for therapeutic use of self", British Fournal of Occupational Therapy, Vol. 81 No. 5, pp. 276-284, doi: 10.1177/0308022617745007.

Kaiser, H.F. (1974), "An index of factorial simplicity", Psychometrika, Vol. 39 No. 1, pp. 31-36.

Kielhofner, G. and Fossey, E. (2006), "The range of research", in Kielhofner, G. (Ed.), Research in Occupational Therapy: methods of Inquiry for Enhancing Practice, F. A. Davis Company, Philadelphia, pp. 29-30.

Knapp, T.R. and Brown, J.K. (1995), "Ten measurement commandments that often should be broken", Research in Nursing E Health, Vol. 18 No. 5, pp. 465-469, doi: 10.1002/ nur.4770180511.

Myers, J.H. (1999), Measuring Customer Satisfaction: how Buttons and Other Measurement Issues, AMA, Chicago (IL).

Nouwen, A., Urquhart Law, G., Hussain, S., McGovern, S. and Napier, H. (2009), "Comparison of the role of self-efficacy and illness representations in relation to dietary self-care and diabetes distress in adolescents with type 1 diabetes", Psychology E Health, Vol. 24 No. 9, pp. 1071-1084, doi: 10.1080/ 08870440802254597.

Opseth, T.M., Carstensen, T., Yazdani, F., Ellingham, B., Thørrisen, M.M. and Bonsaksen, T. (2017), "Self-efficacy for therapeutic mode use among occupational therapy students in Norway", Cogent Education, Vol. 4 No. 1, pp. 1-9, doi: 10.1080/2331186X.2017.1406630. 
Pan, A.W. and Liu, L.-T. (2016), "Therapeutic relationship and treatment outcome", American fournal of Occupational Therapy, Vol. 70 No. S4, p. 7011510234, doi: 10.5014/ ajot.2016.70S1-PO7019.

Punwar, A.J. and Peloquin, S.M. (2000), Occupational Therapy: Principles and Practice, Lippincott Williams \& Wilkins, Philadelphia.

Ritter, V.C., Thørrisen, M.M., Yazdani, F. and Bonsaksen, T. (2017), "Further validation of the norwegian self-efficacy for therapeutic mode use", Occupational Therapy International, Vol. 2017, doi: 10.1155/2017/9745373.

Ritter, V.C., Yazdani, F., Carstensen, T., Thørrisen, M.M. and Bonsaksen, T. (2018), "Psychometric properties of an instrument derived from the intentional relationship model: the Self-Efficacy for recognizing clients' interpersonal characteristics (N-SERIC)", The Open fournal of Occupational Therapy, Vol. 6 No. 2, doi: 10.15453/2168-6408.1423.

Scholz, U., Doña, B.G., Sud, S. and Schwarzer, R. (2002), "Is general self-efficacy a universal construct? Psychometric findings from 25 countries", European fournal of Psychological Assessment, Vol. 18 No. 3, pp. 242-251, doi: 10.1027/10155759.18.3.242.

Scholz, U., Nagy, G., Göhner, W., Luszczynska, A. and Kliegel, M. (2009), "Changes in self-regulatory cognitions as predictors of changes in smoking and nutrition behaviour", Psychology \& Health, Vol. 24 No. 5, pp. 545-561, doi: 10.1080/08870440801902519.

Schwank, K., Carstensen, T., Yazdani, F. and Bonsaksen, T. (2018), "The course of self-efficacy for therapeutic use of self in Norwegian occupational therapy students: a 10-month follow-up study", Occupational Therapy International, Vol. 2018, doi: 10.1155/2018/2962747.
Streiner, D.L., Norman, G.R. and Cairney, J. (2015), Health Measurement Scales: A Practical Guide to Their Development and Use, Oxford University Press.

Taylor, R.R. (2008), The Intentional Relationship: Occupational Therapy and Use of Self, FA Davis, Philadelphia.

Taylor, R.R. (2014), "Therapeutic relationship and client collaboration", in Schell, B., Gillen, G. and Scaffa, M. (Eds), Willard and Spackman's Occupational Therapy, Lippincott Williams \& Wilkins, Philadelphia, pp. 428-429.

Taylor, R.R., Lee, S.W., Kielhofner, G. and Ketkar, M. (2009), "Therapeutic use of self: a nationwide survey of practitioners' attitudes and experiences", American fournal of Occupational Therapy, Vol. 63 No. 2, pp. 198-207, doi: 10.5014/ajot.63.2.198.

Vax, S., Schreuer, N. and Sachs, D. (2012), "Work-related self-efficacy of occupational therapists in mental health", Scandinavian fournal of Occupational Therapy, Vol. 19 No. 1, pp. 42-48, doi: 10.3109/11038128.2010.527366.

Yazdani, F. and Bonsaksen, T. (2019), "Psychometric properties of the self-efficacy for therapeutic mode use tool in a mixed sample of occupational therapists: a pilot study", International fournal of Therapy and Rehabilitation, Vol. 26 No. 6, pp. 1-8, doi: 10.12968/ijtr.2018.0035.

Yazdani, F. and Tune, K. (2016), Self-Efficacy for Therapeutic Use of Self, Oxford Brookes University, Oxford.

Zeegers, P. (2004), "Student learning in higher education: a path analysis of academic achievement in science", Higher Education Research E Development, Vol. 23 No. 1, pp. 35-56, doi: 10.1080/0729436032000168487.

\section{Corresponding author}

Samaneh Karamali Esmaili can be contacted at: samauneh.esmaeili@gmail.com 